\title{
The Style Features of Songs Filled with Ancient Chinese Classical Poetry in the New Era
}

\author{
Li Xue ${ }^{1, *}$ \\ ${ }^{1}$ School of Music, Anshan Normal University, Anshan, Liaoning 114000, China \\ *Corresponding author. Email: 120397292 @qq.com
}

\begin{abstract}
In the new era, with people's pursuit of the integration of classics and fashion, many songs filled with ancient Chinese classical poetry have sprung up like bamboo shoots after a spring rain, and have undergone rapid changes in style. It contains modern people's longing for Chinese traditional culture, and also contains that songs filled with ancient Chinese classical poetry in the new era are awakening the life and growth power of classical poetry with notes. Its unique characteristics of The Times, fashion and inclusiveness perfectly combine singing with literature and art.
\end{abstract}

Keywords: New era, Songs filled with classical poetry, Style.

\section{INTRODUCTION}

Traditional Chinese culture, as the spiritual food to guide the masses, enriches people's cultural life. Under the influence of cultural thoughts in the new era, the Chinese nation has set off a nationwide upsurge of learning Chinese traditional culture, mainly in the study of Chinese classical poetry. With the development of society, people have diversified ways to learn classical poetry, not only through books, but also through watching the wonderful layered programs such as Chinese Poetry Conference, Good Chinese Poetry and Classic Songs spread broadcast in recent years, or listening to audio books, so that the cultural essence of Chinese nation with the breath of the new era can walk into thousands of households, leading all the Chinese nation's descendants to feel the beauty of poetry, sharing the fun of poetry. In order to keep pace with the new era, songs filled with ancient Chinese classical poetry are undergoing unprecedented changes in style. This paper mainly gives examples of the newly created works in "Classical Songs of Circulation". The author believes that the vast majority of works in this program are created with ancient poems as lyrics, which can become the representative of Chinese classical poems and songs in the new era.

\section{THE ERA IS FULL OF FLAVOR}

Songs filled with ancient Chinese classical poetry in the new era adapt to the call of The Times, and endue obscure or obscure poems to music creation. People will see from the program "Classics Live on Forever" that the ancient poems selected by the program group are representative works with universal applicability to the current Chinese society. The lyrics, melody and singing are carefully selected, so that people can better understand the theme and emotion expressed in the poetry.

\subsection{Poetry and Song}

Songs filled with ancient Chinese classical poetry in the new era mainly follow the charm of the poetry itself. The melodic lines of music creation are basically the same as the intonation lines of the poetry. Most of the works have simple notes, slow rhythm and smooth melody lines. In detail, some melodies mimic the lengthening and duration of the last word of each poem, and individual words are added with pronunciation consistent with the intonation of the recitation. The melodic lines of songs such as Guan Ju and Quiet Night Thought were all created with the change of tone of the poems. The emotional ups and downs of the song expressed are consistent with the pitch of 
the song melody. The charm and artistic conception of poetry are better interpreted in music.

\subsection{Popularity of Music}

"Use popular elements to package classics, so that simple poetry can be innovative, so that static Chinese characters into musical notes can linger in the ear." [1] As Hui Man-kwong, deputy director of Integrated Channel, said, "We have brought those timeless classics back to the hearts and minds of contemporary people in the most popular and easily transmitted way." [2] By adding popular melodies to favorite ancient poems and songs and using modern creative techniques, composers have created a variety of singing works of ancient poems and songs, making Chinese classical poems and songs more popular among vocal music lovers and the public, such as Tan Weiwei "Ink Plum", Wang Leehom's "Three Character Classic", Wang Yuan's "Long Song Line", Huang Ling's "Soul of Chu", Jike Junyi's "Send to the Fortress" and so on.

\subsection{Modernity of Lyrics}

In terms of the use of lyrics, a small part of the Chinese songs filled with classical ancient poetry created in the new era are the original works without any changes, and most of the works are recreated on the basis of the original poetry, extending part of the lyrics. This part of the extended lyrics is what people fill in after understanding the poetry in the current era. There are many modern language descriptions of the poetry, giving modern thoughts, letting the poetry of thousands of years have the breath of the new era, and making it convenient for people to feel the emotion of the original work. For example, Zhang Jie sang "Hard Roads in Shu", which was written by Zhang Jie himself, reflecting the down-to-earth and hard-working spirit of Bashu people.

\section{FASHION AND CLASSIC COEXIST}

In order to achieve the proper fusion of classic and fashion, and to continue the inheritance of heavy poetry, creative expression of classical poetry and songs suitable for the needs of the new era was born. Songs filled with ancient Chinese classical poetry in the new era not only make bold innovations in melody creation, but also boldly practice the collision between fashion and classic in stage design, lighting transformation and clothing selection, bringing people all-round audio-visual enjoyment and enhancing the communication effect.

\subsection{Star Support}

In today's society, stars can represent many products and promote many businesses. Stars themselves are the symbol and carrier of fashion in today's society. With the help of stars, the propagation speed and effect of Chinese classical poetry in the new era will be greatly enhanced. In the new era, songs filled with ancient Chinese classical poetry were sung by film and television stars Such as Wang Yaoqing, Wan Qian, Wu Jinyan and Xiao Zhan. There are Li Guyi, Zhou Bichang, Sha Baoliang, Li Jian, Wang Yuan and other very famous singers of all times.

\subsection{Dazzling Stage}

In order to give people a deeper understanding of the song, the stage and lighting are used in the use of mind, using the transformation of space, mirror design, star design, sea design, and dream design, presenting a multidimensional visual effect. It's refreshing. When Dong Baoshi sang "Joining the Army", all kinds of one-to-one armors were put on the stage to support him, giving people an immersive feeling. When You Zhangjing sang "Shu Xiang", the folding screen, calligraphy and painting all showed the literary atmosphere of Temple of Marquis Wu. When Shang Wenjie sang "Cang Huang", she stood in the middle of the red long sleeves, really like a flying Apsaras. When Xiao Zhan sang "Bamboo and Stone", there were clusters of bamboos on the stage, giving people a sense of tenacity and upward strength. When the family of Sha Yi and $\mathrm{Hu} \mathrm{Ke}$ sang "Wind", the scenery of regions south of the Yangtze River with small bridges over the flowing stream on the stage gave people a fresh feeling. When Lin Zhixuan sang "Chang Xiang Si", the stage backdrop was Chang'an City. Through the colorful Chang'an City with multi-culture and then to the black and white Chang'an City in the dream, different colors were used to restore the poet $\mathrm{Li}$ Bai's homesickness. When Shang Wenjie sang "Song of White Snow", the stage presented a sci-fi snowflake kingdom, making the harsh frontier fortress a romantic and picturesque fortress, with the verve and spirit of the Tang Dynasty. 


\subsection{Exquisite Costumes}

In order to enhance the effect of the work, each singer carefully chose clothes suitable for singing poetry works. Huang Ling wore sleeves symbolizing armor for her song "Soul of Chu", and Zahi Bing Zo and Alan Dawa Dolma wore beautiful Tibetan costumes for their song "Desert Sand Like Snow". The Band also wore elaborate Mongolian costumes. There are also many actors wearing costumes to meet the situational requirements of the work.

\subsection{Strong "Chinese Style"}

"The so-called 'Chinese style' is a style with elements of Chinese characteristics". Having "Chinese style" is one of the main characteristics of the creation of songs filled with ancient poetry, such as Gong Linna's Twenty-four Solar Terms Concert of Classical Poetry. Gong Linna used her superb singing skills, spanning several vocal ranges, using the conversion of different timbres, and vividly showing the pictures of the twenty-four solar terms in a year in front of people. Among them, "Seek Truth Continually" was accompanied by a magnificent chime, which is the most representative musical instrument of ancient China. Other works were also accompanied by bamboo flute, Guzheng, Xun, Xiao and other national musical instruments, making people deeply intoxicated. There were also many works in "Classics Live on Forever" that were accompanied by Pipa, Horse-head Lute, Sarangi and so on. In particular, Dai Quan's "Lyrics of Autumn Wind" was accompanied by Bili and Guqin, showing the strong Chinese style. "Quiet Night Thought" sung by Lei Jia, "Dynasty Songs" and "Ode to the Plum Blossom" with Peking Opera clips of "Plain Tune" sung by Wang Hengyi and Zhou Zhaoyan, the "Ode to the Pear Blossom", "Ink Plum", "Ode to the Plum Blossom", and "Peony Pavilion" sung with the arias of opera,"Bie Jun Tan" created in the tone of Shaanxi dialect and "Drunken in Chang'an" with strong Shaanxi flavor, "The Desert Sand Is Like Snow" sung in Tibetan language and integrated with Tibetan folk tunes, "Waves Washing the Sand" integrated with Qinghai folk song "Flower", "Chile Song" and "The Grass Is Far Away at Sunset" with Mongolian prairie charm, etc., all are full of Chinese style. "The Peach Blossom Fan" sung by Wang Sulong and Zhang Jun combined Kun Opera with pop music, making the soft Kun Opera exude vitality. The beauty of its singing, costumes, and stories all reflected a strong Chinese style. Wang
Leehom's "A Bridge of Fate" combines the national pentatonic mode of "Gong, Shang, Jue, Zhi, Yu" and four tones of "Yin, Ping, Shang, Qu" to create glissando to reflect the Chinese style. Huan Qing's "Ye Yu Ji Bei" integrates traditional Chinese folk music and intangible cultural heritage music Bimo music into the work to reflect the Chinese style.

\section{BEING INCLUSIVE}

In the new era, songs filled with ancient Chinese classical poetry present diversified forms. Compared with the songs filled with ancient poetry created by masters such as Huang $\mathrm{Zi}$ and $\mathrm{Shi}$ Guangnan, which were sung in the last century with the bel canto and piano accompaniment, in the new era of cultural diversity and artistic diversity, people's needs have become the driving force for the diversified creation of songs filled with ancient poetry, as well as the driving force for diversified singing. The expression of the work is more inclusive.

\subsection{The Integration of Musical Instruments}

From the composition of the band to the selection of musical instruments, a variety of fusions have been carried out. For example, Zhong Zhentao sang "Yujiaao • Autumn Thought", combining suona and electric guitar. The desolate picture and regional style presented by the suona and the inner emotions that the guitar wanted to express made people truly feel homesickness and patriotism. Wu Tong's song "All Over the River Red" is also a combination of folk Suona and rock band, giving people a feeling of thousands of troops and horses. His performance of "A Short Song Ballad" also integrated the musical instruments Sheng and piano, which connected people and nature, to reflect a kind of simple and plain beauty. Another example is "Sunset, Far Grass" sung by Haya Orchestra. The combination of morin khuur and piano, originally two different musical instruments, creates an excellent audio-visual experience. When Nan Sun sings the Song of the Wind, Jinlong Fang combines the lost five-string pipa with the modern band, showing the fierce scene on the battlefield, which has both the sense of desolation and the regret of unfulfilled ambition. Huan Qing's "Ye Yu Ji Bei" used the mysterious jew's harp, Moon Lute and modern musical instruments to show a quiet picture in front of the audience, giving people a very simple feeling. Chen 
Weilun's "Iron Horse and Autumn Wind" boldly uses electronic music to harmonize with Guqin and $\mathrm{Gu} \mathrm{Se}$, without any feeling of strangeness, fully showing the tension of ancient Chinese poetry.

\subsection{The Fusion of Singing}

Dong Baoshi sang "River Snow" with rap. He thought: "Rap and ancient poetry have similarities. They both pay much attention to rhyme and rhythm, especially classical ancient poetry. From this, people can conclude that ancient poetry is the basis of rap in terms of the beauty and rhythm of words, and creators can draw inspiration for creation from poetry. This also ensures that the rap language is smoother and more poetic during singing. Through the singing form of rap, Dong Baoshi shows people the image and impact of the poem River Snow and the beauty of looking down the lens that this poem can create, and then dissociates people out. In fact, it tells people that they need a kind of tenacity and a kind of courage to break through themselves. Zhang Jie's "Hard Roads in Shu" uses Sichuan dialect to rap, integrates with Sichuan high tune, and also uses vocal accompaniment to express the spirit of roads in Shu incisively and vividly. Liao Changyong and Liao Minchong, father and daughter, sang "Impromptu Lines Written on a Spring Day", which combined popular singing and bel canto with piano accompaniment, and the fresh and beautiful spring scenery showed the dialogue spanning time and space. When singing "Rolling Waves of the Long River Flow Toward the East", Yang Hongji began with the spoken parts of the opera. And during the singing, he added the glissando and vibrato from the opera and combined the bel canto with the opera singing, making his singing closer to the hearts of the Chinese people. Tan Weiwei's "Ink Plum" combines with popular singing to produce an opera song, giving people a sense of space and intimacy. The HAYA's "The Grass Is Far Away at Sunset" presents the idyllic melody, allowing the audience to feel the cultural fusion of the South and the North. Among them, popular singing and Mongolian representative singing method Khoomei are integrated. The Mongolian music that is healing, simple and natural is added with the rhythm of Sauurdin, reflecting that the Mongolian people are a nation that loves dance, music and life. "Ode to the Pear Blossom" sung with opera, "The Peach Blossom Fan" integrated with pop music and opera, "Ode to Gallantry" sung by rap, and "Quiet Night Thought" sung by Lei Jia with folk style singing all break the pure piano accompaniment of songs filled with ancient poetry and only sung by musicians.

\subsection{The Fusion of Forms}

"When an ancient poem meets a Chinese cartoon, when an ink painting meets a nursery rhyme." [3] This is a series of public welfare animations produced by the vocal animation team. Director Peng Qingzheng interspersed the ancient poems and songs during the animation playing on the stage of "Classical Songs and Traditions", and conveyed the classic works with the songs in the animation works. They incorporated ancient Chinese poems into every detail of the animation, presenting a warm and beautiful Oriental scene. For example, Yuanri is a work that uses the eyes of a child to see the traditions and customs of the ancient Spring Festival. The verses "The crepitation of firecrackers sends off the old year. The spring breeze brings warmth into thousands of households" depict the form of New Year's ballads in memory. It allows children to see more Chinese elements in the process of watching cartoons. The image of the work is exquisite and the traditional landscape painting is vivid in color matching. This enables some traditional Chinese culture to be more down-to-earth, fashionable, and younger, and no longer make it too high to be popular. It can be understood by everyone, being suitable for people of all ages. The holographic projection technology allowed Teresa Teng to stand on the stage again and perform a special form of chorus "May We All Be Blessed with Longevity" with the little girl Liu Runtong, transcending time and space to realize the dream of singing the classics of Teresa Teng, making people even more moved in a trance.

\subsection{The Integration of Singers}

Many famous stars and celebrities are invited to sing, and the singers are also mixed. The singers range from young children to people in their 80s. The most touching thing is the Silent Choir composed of deaf teenagers. They went through all kinds of difficulties to get the children to stand on the stage and sing "Paint Silent", opening the door of confidence for the children and letting them find the light to open the door to the outside world, which is every "ah" they uttered. They sing a hymn to life, a wordless poem. From Xia Hongpeng, a criminal police with terminal cancer, to Chen Xiaolong from Laos, Sally from Cambodia, Rulan from Thailand, You Zhangjing from Malaysia, Kristin, Happy Rogers, and Beeland Rogers Sisters 
from the United States and other international friends, from Phoenix Legend, Four Heroes of the East, The Wild Child Choir, Gushi Tongyun Choir, Chinese Academy of Sciences Veteran Scientist Choir, and Guizhou Yunshang Dong Village Choir, to the Inheritor of Intangible Cultural Heritage of Daliang Mountain in Sichuan, and from children, students, teachers, police officers, hosts, singers, actors, and scientists to cartoon directors, singing has crossed national boundaries and occupations.

\section{CONCLUSION}

Songs filled with ancient Chinese classical poetry in the new era integrate all possible art forms. The integration of such a culture keeps Chinese culture alive, developing and advancing. It records people's views on nature, on life, on emotion. When you appreciate it, it seems that you are talking to the poet in space and communicating with him. You feel that your imagination has been expanded in your hearing. Songs filled with ancient Chinese classical poetry are opening up people's historical feelings in the new era. It will continue to innovate with the rapid development of The Times, and it will continue to create new poems with people's expectation and pursuit.

\section{AUTHORS' CONTRIBUTIONS} Xue.

This paper is independently completed by $\mathrm{Li}$

\section{REFERENCES}

[1] Keke Lian. Song Category Analysis of "Classics Forever Circulating" [J]. Contemporary Music,2021.04

[2] Xinlu Lu. Modernity Research of Chinese Classical Poetry and Songs [J]. Art Education,2019.01

[3] Mei Tian. "Classics Endure forever" and poetry to song, so that the classics revitalize again [J]. Television Research,2018.06

[4] Rong Yi. Thoughts on re-interpreting Chinese classical Poetry and art songs with modern vocal music Consciousness [J]. Curriculum Education Research,2019.07

[5] Hongtao Wang. On the Research assumption of Chinese classical Poetry and art song from the perspective of language musicology [J]. Northern Music, November 2018
[6] Chang Liu. On Integrating Chinese Classical Poetry into Vocal Music Teaching in Colleges and Universities [J]. Art Education,2021.07 